\title{
A Note Concerning the Corrigendum to "On the Non-Uniform Propagation of Weak Discontinuities in Magnetogasdynamics"
}

\author{
Volume 78, pages 397-398 (1982)

\section{D. Sharma \& Radhe Shyam}

The discussion in the above corrigendum (by Professors RAMA SHANKAR and MOHAN PRASAD) has been taken almost verbatim and without due acknowledgement from page 41 of our paper "Growth and decay of weak discontinuities in radiative gasdynamics" which appeared in Acta Astronautica 8, pp. 31-45 (1981).

In the following references, we have obtained some further general results which can be used for a more satisfactory discussion than in the corrigendum.

1. V. V. Menon \& V. D. Sharma: Correction to a paper of Ram concerning the behaviour of the solution of Bernoulli equation, Il Nuovo Cimento 58B, pp. 64-72 (1980).

2. V. V. Menon \& V. D. Sharma: Characteristic wave fronts in magnetohydrodynamics, Journal of Mathematical Analysis and Applications 81, pp. 189-203 (1981).

3. V. V. Menon, V. D. Sharma \& A. Jefrrey: On the general behaviour of acceleration waves, to appear in Applicable Analysis.

$$
\begin{aligned}
& \text { Applied Mathematics Section } \\
& \text { Institute of Technology } \\
& \text { Banaras, Hindu University } \\
& \text { Varanasi, India }
\end{aligned}
$$

(Received July 27, 1983)

The editors of the Archive for Rational Mechanics and Analysis wish to thank Professors Sharma and SHYAm for calling their attention to this matter. 